\title{
HyperCast: Hyperspectral satellite Image Broadcasting with Band Ordering Optimization
}

Ahmed Hagag ${ }^{a, b}$, Xiaopeng Fan ${ }^{a^{*}}$ and Fathi E. Abd El-Samie ${ }^{c}$

${ }^{a}$ Department of Computer Science and Technology, Harbin Institute of Technology, Harbin 150001, P.R. China.

${ }^{b}$ Department of Information Technology, Faculty of Information Technology,

Egyptian E-Learning University, Dokki, Giza, 12611, Egypt.

${ }^{c}$ Department of Electronics and Electrical Communications, Faculty of Electronic Engineering, Menoufia University, Menouf, 32952, Egypt.

E-mails: ahagag88@gmail.com, fxp@hit.edu.cn,fathi_sayed@yahoo.com

Phone: $+8618845079721,+8615045688860,+201061257233$

(*Send correspondence to Xiaopeng Fan)

E-mail: fxp@hit.edu.cn

Telephone: +8615045688860

This work was supported in part by the National Science Foundation of China (NSFC) under grants 61472101, 61631017 and 61390513, the Major State Basic Research Development Program of China (973 Program 2015CB351804), and the National High Technology Research and Development Program of China (863 Program 2015AA015903). 


\title{
HyperCast: Hyperspectral Satellite Image Broadcasting with Band Ordering Optimization
}

\begin{abstract}
This paper presents a novel framework for hyperspectral satellite image broadcasting over wireless channels. We present a new hyperspectral band ordering algorithm that improves the compression performance. The proposed scheme employs the 1D low-complexity Karhunen-Loève transform (KLT) that uses a clustering approach for spectral decorrelation. After that, the 2D DCT is applied to remove the redundant information from the spatial bands. The DCT components are quantized using a simple DCquantization algorithm. After that, the transmission power is directly allocated to the quantized data according to their distributions and magnitudes without forward error correction (FEC). These data are transformed by Hadamard matrix and transmitted over a dense constellation. Experiments demonstrate that the proposed scheme improves the average image quality by $6.98 \mathrm{~dB}$ and $3.48 \mathrm{~dB}$ over LineCast and SoftCast, respectively, and it achieves up to $6.14 \mathrm{~dB}$ gain over JPEG2000 with FEC.
\end{abstract}

Keywords: Hyperspectral satellite images; image broadcasting; Karhunen-Loève Transform (KLT); hyperspectral band ordering; SoftCast; LineCast; wireless communications.

\section{Introduction}

Hyperspectral images have been widely used for a large number of applications in remote sensing. This type of images represents the reflected solar radiation. Hyperspectral images are generated by collecting hundreds of bands representing the same area of the earth surface in different spectral sampling intervals. The data produced can be viewed as a cube, as shown in Fig. 1, having two dimensions that represent the spatial position and one that represents the wavelength. However, at the time we gain high resolution spectral information, we generate massively large image data sets. Therefore, storage and transmission of this amount of data has become one of the greatest challenges. Several satellite image compression techniques have been presented in [1] aiming to reduce the image size and improve user interaction with the information. 


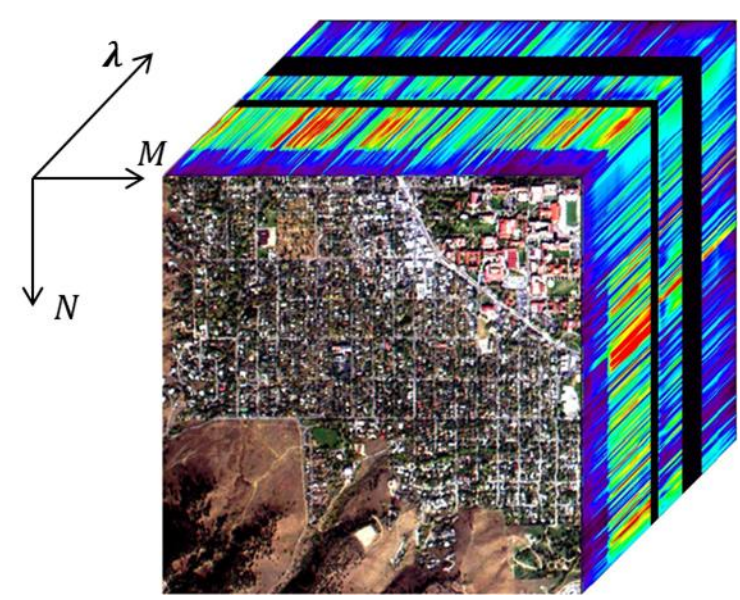

Fig. 1. Hyperspectral image data. The 3-D structure.

Today, hyperspectral satellite images are used in a wide variety of applications and remote sensing projects ranging from independent land mapping services to government and military activities. In agriculture, the need for observational data, aircraft, and satellite remote sensing plays an important role in farm management due to the large volume of information represented in the satellite images. Moreover, for meteorologists, satellite images play an essential role to explore water vapor, cloud properties, aerosol, and absorbing gases. Some of these applications and projects desperately need wireless communications to transmit satellite images. In [2], the research described an experiment to prototype a new combination of satellite, wireless, and terrestrial networking. There is a need for more studies in the future satellite wireless communications. Transfer and analysis of satellite images after the flight may be delayed for days or even weeks, after which it could be found that the photos taken were flawed or incorrect. To overcome this challenge, the research in [3] presented a new project by creating a wireless network system that shortens the time span and streamlines between imagery data collection and end-user applications. Other applications of Geology, Nature, Environment, and Space sciences exist for satellite images in different projects, and we predict the urgent need for using wireless communications in the future.

Now, wireless communication tools are able to transfer large packets of data quickly and efficiently over distances. Shannon concluded two main issues are required to transmit data over wireless channels: source coding (data compression) and channel coding (forward error correction (FEC) and modulation scheme) $[4,5]$. This conventional scheme with separate source coding and channel coding is based on Shannon's source-channel separation principle, and it is the most classic digital scheme. Source coding is designed independently of channel coding. Using this classical scheme, the source data can be transmitted without any loss of information, if the channel is pointto-point (i.e., unicast communication). The channel quality is known or can be easily measured at the source, by the selection for the optimal transmission rate for the channel and the corresponding FEC and modulation [5]. However, for digital broadcast/multicast, a cliff effect challenge is found, where each receiver observes a different channel quality. Thus, the bitrate selected by a conventional wireless image delivery scheme cannot fit all receivers at the same time. If the image is transmitted at a high bitrate, it can be decoded only by those receivers with better quality channels, but it is not reasonable for receivers with worse quality channels. On the contrary, if it transmits at a low bitrate supported by all receivers, it reduces the performance of the receivers with better quality channels, and it is not optimal for performance. Therefore, designing a separate wireless frontend is not the 
best solution for channels having various qualities. In order to overcome the cliff effect, many researchers propose different joint source-channel coding (JSCC) frameworks for distributed image/video transmission [6, 7]. Except for these JSCC works, the transmission of distributed coded image/video is still similar to that of the conventional scheme. In contrast to the separate design, there are many joint image/video coding and transmission schemes that have been proposed for wireless image/video multicasting. SoftCast [8] is one of the analog approaches that are designed within the JSCC framework. The SoftCast encoder consists of the following steps: 3-dimensional discrete cosine transform (3D DCT), power allocation and direct dense modulation. Recently, a linebased coding and analog-like transmission is proposed in [9], called LineCast, which consists of three steps, reading the image line by line and the decorrelation of each line by DCT, scalar modulo quantization, and finally power allocation and transmission.

In this paper, we propose a new wireless coding and transmission scheme, called HyperCast, for broadcasting of hyperspectral satellite images to a large number of receivers. In contrast to the previous separate design, the proposed HyperCast scheme is able to broadcast a satellite image of different resolutions to fit various devices with different display resolutions. HyperCast avoids the cliff effect found in the digital broadcasting schemes by using linear transform between the transmitted image signal and the original pixels luminance. In the proposed scheme, the transform coefficients are directly transmitted through a dense constellation after allocating a certain power without FEC or digital modulation. In multicasting, each user can optimal quality matching for its channel conditions. When the channel SNR increases, the reconstruction PSNR increases accordingly, and vice versa.

Most satellite images have more than one band. These bands contain spectral redundancies. Spectral redundancy means that the information content of one band can be fully or partly predicted from the other bands in the data. LineCast does not exploit the spectral correlation between bands. Therefore, this will lead LineCast to be inefficient with satellite images containing more than one band. In conclusion, LineCast is able to provide better performance than the state-of-the-art 2D broadcasting schemes because of its high efficiency and flexibility of line prediction. However, without a spectral decorrelator, it is still not efficient enough for the use of 3D satellite images. In order to take advantage of both spatial and spectral redundancies found in hyperspectral images, the proposed HyperCast adopts band ordering and grouping algorithms followed by a 3D transform to remove redundancies and improve the compression performance. SoftCast uses the original band/frame ordering, and thus, it is inefficient in the removal of spectral redundancy. Furthermore, the 3D DCT transform of a group of images (i.e., group of bands) is insufficient for removing most of the redundancy in the satellite images. In contrast to SoftCast, the proposed HyperCast adopts a band ordering algorithm for the satellite images. Furthermore, it adopts a hybrid 3D transform based on 1D low-complexity Karhunen-Loève transform (KLT) with a clustering approach [10] as a spectral decorrelator and 2D DCT as a spatial decorrelator. Experimental results on the selected hyperspectral datasets demonstrate that the proposed scheme improves the average image quality by $6.98 \mathrm{~dB}$ and $3.48 \mathrm{~dB}$ over LineCast and SoftCast, respectively, and it achieves up to a $6.14 \mathrm{~dB}$ gain over the conventional scheme.

The rest of the paper is organized as follows. Section 2 reviews some related works. Section 3 describes the proposed HyperCast scheme including both encoder and decoder. Section 4 
discusses the proposed hyperspectral band ordering optimization. The evaluation environment and the experimental results are presented in Section 5. Section 6 gives the concluding remarks of the paper.

\section{Related Works}

\subsection{Digital Broadcasting}

Satellite broadcasting systems separate source coding and channel coding based on Shannon's source-channel separation theorem [4] and [5]. In the source code, there are many techniques that have been recently used to compress hyperspectral images [1]. In channel coding, FEC and digital modulation are used. However, we cannot accommodate all channel conditions simultaneously for all receivers in the broadcasting scenario such that the transmission rate adapts to the actual channel conditions by adjusting the channel coding rate and modulation.

Previous research on satellite image compression concluded that JPEG2000 [11] (waveletbased algorithm) gives better results than others. The research in $[12,13]$ showed that, for lossy compression of satellite multispectral images, JPEG2000 (wavelet-based algorithm) produces better results than others. Also, the research in [1] compared several techniques with different compression methods. All of these methods are wavelet-based algorithms and they showed that the results of these methods are better than other methods. Moreover, the research in [14] showed that, for lossy compression of satellite hyperspectral images, using discrete wavelet transform (DWT) and principal component analysis (PCA) provided improved compression quality when compared with others. In order to improve the performance of the digital broadcasting process, some layered digital schemes consisting of layered source coding and layered channel coding have been proposed $[15,16]$ and [17] such as H.264/SVC [18] with hierarchical modulation (HM) [19] and multi-resolution coding [17]. Although the layered digital scheme has a better performance than the separation coding scheme, it is unable to adapt for users with different classes of channel conditions. H.264 is DCT based algorithm. H.264 coder achieves better performance than JPEG2000 for video coding, because there is a motion between video frames. However, hyperspectral images are generated by collecting hundreds of bands representing the same area of the earth surface in different spectral sampling intervals. Therefore, JPEG2000 is still the best lossy compression method used for the satellite image. The consultative committee for space data systems (CCSDS) developed framework [20] is also a good choice for satellite image compression, however JPEG2000 is more efficient spatially for lossy compression due to the spectral decorrelation whatever by DWT or PCA. In the conventional framework, JPEG2000 with PCA in the spectral decorrelator is used in the digital source coding.

\subsection{SoftCast}

Different from digital broadcasting, SoftCast scheme [8] is an alternative design for wireless image/video streaming. SoftCast is one of the analog approaches designed within a JSCC framework. This scheme can optimize the received distortion by a linear combination of the original signals, which are then directly transmitted over a dense constellation. SoftCast transmits a linear transform of the image/video signal directly over an analog channel without quantization, entropy coding, or 
FEC. This principle naturally enables a transmitter to satisfy multiple receivers with diverse channel qualities.

The SoftCast encoder consists of 3D DCT, power allocation, Walsh-Hadamard transform (WHT), and direct delivery to the wireless physical layer (PHY). The 3D DCT allows SoftCast to remove redundant information within an image/video frame or across frames, while maintaining linear behavior. Power allocation minimizes the total distortion by optimally scaling the DCT components according to their entropy. SoftCast assigns the scaled DCT values to packets. It employs a linear Hadamard transform to make packets of equal power and equal importance. After that, the packets are delivered directly to the PHY, which interprets their data as digital signal samples to be transmitted. At the receiver, for each received packet, the PHY returns the list of coded DCT values in that packet. To reconstruct the signal, SoftCast uses a linear least squares estimator (LLSE) to invert the effect of power allocation and Hadamard transform. Once the decoder has obtained the DCT coefficients, it can reconstruct the original image/video frames by taking the inverse of the 3D DCT. Almost all the steps in Softcast are linear, and thus the channel noise is directly transformed into reconstruction noise of the image/video. Therefore, it is not required to know the users' channel conditions, and each receiver can naturally get a quality matching its channel conditions.

\subsection{LineCast}

LineCast is an up-to-date satellite image delivery scheme [9], which depends on line-based coding and analog-like transmission. The line-based framework provides a natural way to avoid broadcasting of the whole areas of images for all users. In the analog-like transmission, every scanned line of an image is compressed by a transform-domain scalar modulo quantization without prediction to significantly reduce computations at the satellite. Furthermore, the quantized coefficients are directly transmitted without syndrome coding or channel coding similar to SoftCast. Every user can receive the image with a quality matching its channel conditions. LineCast is mainly utilized in the broadcasting of satellite images.

The LineCast encoder consists reading the image line by line, decorrelating each line by a 1D DCT, scalar modulo quantization, power allocation, and transmission. To decorrelate each line, LineCast uses 1D DCT, and then a scalar modulo quantization is performed on the DCT coefficients with the same technique used in distributed source coding (DSC) [21], which partitions source space into several cosets and transmits only coset indices to the decoder. After the scalar modulo quantization, a power allocation technique is employed to minimize the total distortion. Similar to SoftCast, LineCast employs linear Hadamard transform to achieve resilience to packet losses. After that, the data is directly mapped into the wireless symbols by a very dense 64K-QAM without FEC or modulation. At the receiver, LineCast uses LLSE to reconstruct the line signal from the DCT coefficients. Moreover, side information is generated to aid the recovery of transform coefficients. Finally, a minimum mean square error (MMSE) technique is used to denoise the reconstructed signal. 


\section{Proposed HyperCast Scheme}

The proposed HyperCast scheme transmits a hyperspectral satellite images over the OFDM channel directly without FEC or digital modulation. Figure 2 shows the framework of the proposed HyperCast scheme.

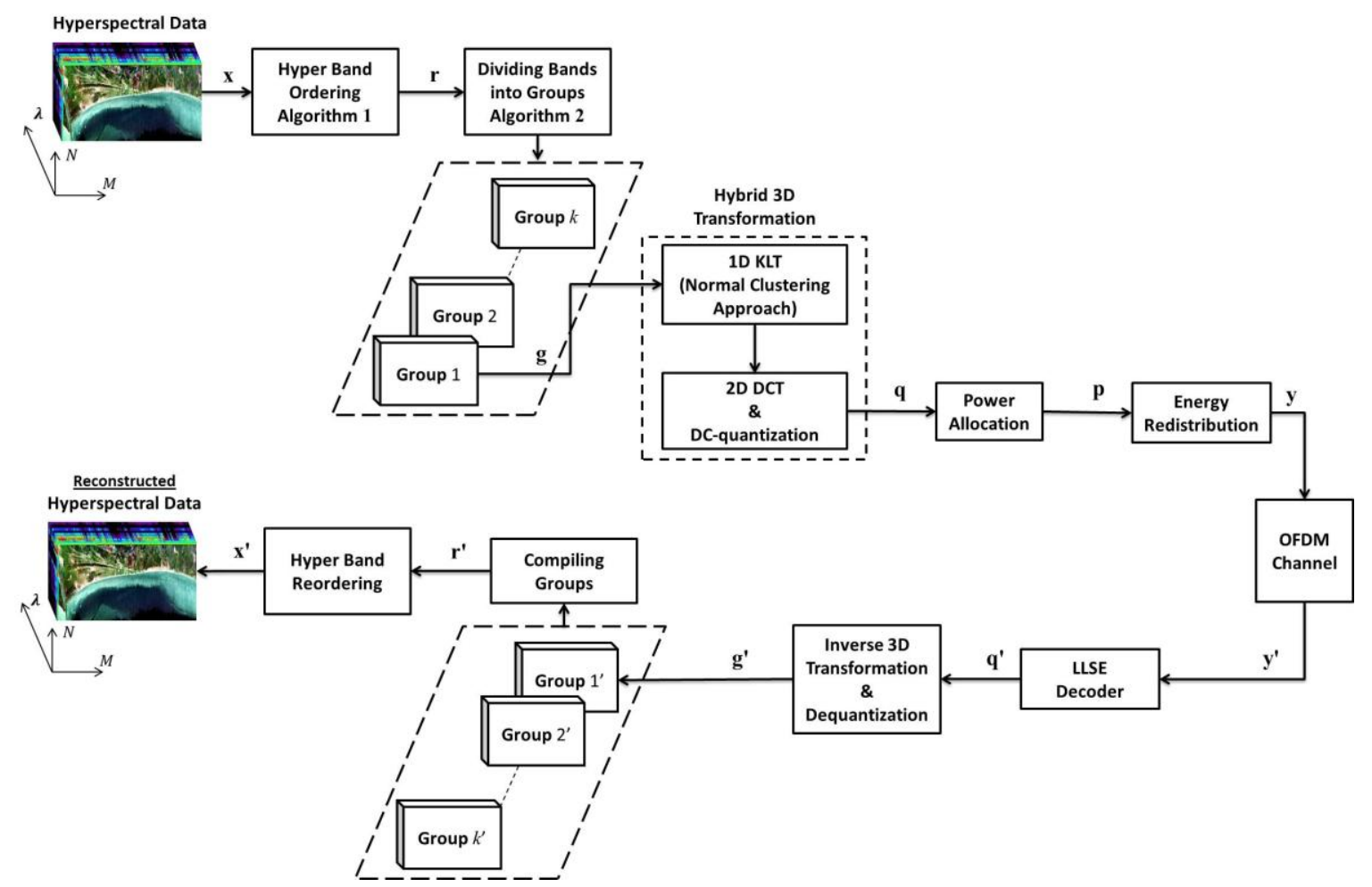

Fig. 2. Framework of the proposed HyperCast scheme with band ordering.

At the encoder, the HyperCast scheme begins with band ordering to exploit the spectral redundancy as will be explained in Section 4. After that, it divides the input ordered hyperspectral image $\mathbf{r}$ into groups of bands $(\mathrm{GOB}) \mathbf{g}=\left\{g_{1}, g_{2}, \ldots, g_{k}\right\}$, where $\mathbf{g}$ contains $k$ groups. Each group contains I bands, and the 1D low-complexity KLT transform is performed on each group and followed by the 2D DCT to remove redundant information within each band as well as across spectral dimension for each group. The DCT are then quantized with a proposed DC-quantization scheme. Owing to the fact that the outputs q's have different distributions, a power allocation technique is employed to every $\mathbf{q}$ to protect it from channel noise. Before transmission, HyperCast employs a Hadamard transform to redistribute energy as used in communication systems. Finally, the weighted vector $\mathbf{y}$ is transmitted through the OFDM channel without FEC or digital modulation.

At the decoder, after the PHY returns the list of coded DCT vector ' $y^{\prime}$, the LLSE is applied to provide a high-quality estimate of the reconstructed DCT coefficients vector ' $\mathbf{q}$ '. Once the decoder has obtained $\mathbf{q}^{\prime}$, it can reconstruct the original groups by DC-dequantization and then taking the inverse of the 2D DCT, followed by the inverse KLT. After that, HyperCast combines the reconstructed groups $\mathbf{g}^{\prime}=\left\{g_{1}{ }^{\prime}, g_{2}{ }^{\prime}, \ldots, g_{k}^{\prime}\right\}$ to recover the hyperspectral data vector $\mathbf{r}^{\prime}$. Finally, the hyperspectral band reordering is applied to reconstruct the whole hyperspectral image in $\mathbf{x}^{\prime}$. In the following subsections, each aspect of the proposed HyperCast scheme is described. 


\subsection{Band Ordering and Grouping}

Before the 3D transformation, the satellite image $\mathbf{x}$ is first ordered and grouped by a proposed algorithm based on the mean value and the mean square error (MSE) between bands. The detailed process will be presented in Section 4.

$$
\begin{gathered}
\mathbf{r}=\operatorname{order}(\mathbf{x}) \\
\mathbf{g}=\operatorname{divide}(\mathbf{r})
\end{gathered}
$$

where $\mathbf{g}=\left\{g_{1}, g_{2}, \ldots, g_{k}\right\}$, and each group $g_{u}$ contains $/$ bands for $u=1,2,3, \ldots, k$.

\subsection{D Transform and Quantization}

The KLT achieves good performance in hyperspectral image compression [22]. However, the high complexity of this transform is the largest challenge. Different research teams have presented several proposals to lessen the computational complexity problem [10, 23, 24, 25, 26] and [27]. Performing KLT over all bands will be more efficient. However, it will be more complex than the division strategy used in the proposed framework. Moreover, it requires the encoder to send a large amount of metadata to the decoder to assist it in inverting the received signal. Therefore, we divide the hyperspectral bands into groups to achieve a lower encoding complexity as shown in [30]. Another reason is to reduce the amount of metadata.

In [23], the divide-and-conquer spectral decorrelation strategy for hyperspectral image processing is presented and compared with other strategies. In the HyperCast scheme, we use the divide-and-conquer strategy presented in [10] and [27]. This strategy implements a normal clustering approach to reduce the computational complexity of KLT, called single-level [23]. This strategy works well when the spatial dimensions are small. Therefore, it works well in the proposed scheme, because we divide the hyperspectral images into groups; every group contains a small number of bands, which is set to 4 in our implementation. As shown in Fig. 3, for each group $g_{u}$, we apply a 1D KLT (single-level strategy) to remove redundant information within a spectral dimension. After that, we adopt a 2D DCT for every band as a spatial decorrelator, and quantize the DCT coefficients by a simple DC-quantization.

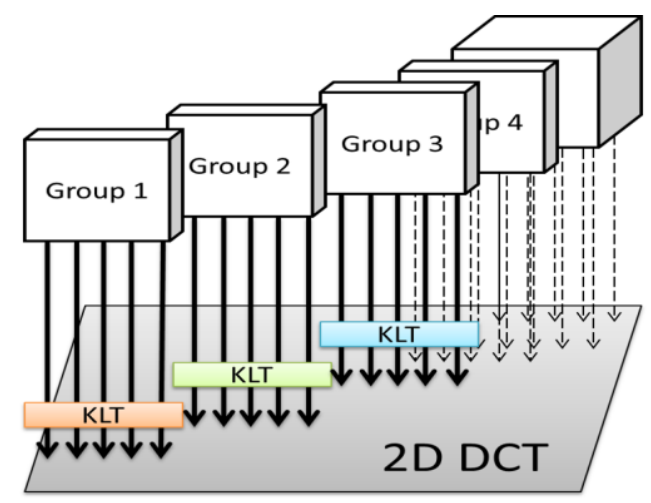

Fig. 3. Illustrative diagram of single-level KLT transform with clustering approach. 


\subsection{1. $1 D K L T$}

After band ordering and grouping, we perform a simple pre-processing by subtraction of the integer mean value $m_{u}$ from each group $g_{u}$. The purpose of this subtraction is to reduce the band energy. Therefore, the transmission power will be small and the KLT transform is given by

$$
\begin{gathered}
K_{u}=\mathbf{K L T}_{\Sigma_{g_{u}}}\left(g_{u}-m_{u}\right)=\mathbf{V}_{u}^{\mathbf{T}}\left(g_{u}-m_{u}\right), \\
\text { and } m_{u}=\left|\operatorname{mean}\left(g_{u}\right)\right|_{\text {Round }}
\end{gathered}
$$

where $\Sigma_{g_{u}}$ is the covariance matrix of $g_{u}$, and $\mathbf{V}_{u}$ is the orthogonal KLT transform matrix for the group $g_{u}$. The orthogonal matrix $\mathbf{V}_{u}$ is obtained from the singular value decomposition (SVD) of the covariance matrix $\Sigma_{g_{u}}$ of the whole set of input data group $g_{u}$.

Note that each group $g_{u}$ contains $N$ rows, $M$ columns, and $I$ bands. For each group $g_{u}$, the mean value $m_{u}$ and the transform matrix $\mathbf{V}_{u}$ need to be transmitted to the receiver as metadata so that $g_{u}$ can be recover, correctly.

\subsection{2. $2 D D C T$ and $D C$-quantization}

We divide each hyperspectral image to $k$ groups. Each group $u$ contains I bands. For each band, the input is a set of $t=M \times N$ data values $p_{u}[t]$ (KLT components), and the output is a set of $t$ DCT transform coefficients $d_{u}[t]$.

$$
\begin{aligned}
& d_{u}[i, j]=\sqrt{\frac{2}{M}} \sqrt{\frac{2}{N}} C_{i} C_{j} \sum_{x=0}^{N-1} \sum_{y=0}^{M-1} p_{u}[x, y] \cos \left[\frac{(2 x+1) i \pi}{2 N}\right] \cos \left[\frac{(2 y+1) j \pi}{2 M}\right], \\
& \text { for } 0 \leq i \leq N-1 \text {, and } 0 \leq j \leq M-1 \text {. and } \\
& C_{f}= \begin{cases}1 / \sqrt{2} & \text { for } f=0 \\
1 & \text { for } f>0\end{cases}
\end{aligned}
$$

where $u$ refers to the group index, and $u=1,2, \ldots, k$. The coefficient with zero frequency of both dimensions is called the direct current (DC) coefficient, and the other coefficients are called alternating current (AC) coefficients, as shown in Fig. 4. 


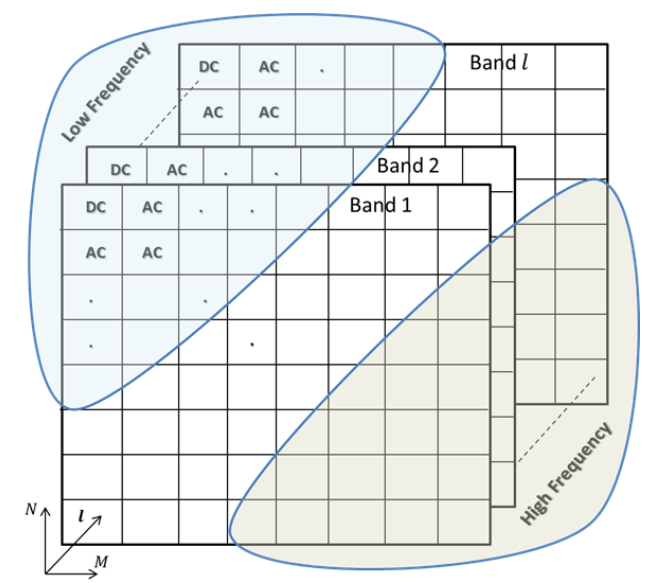

Fig. 4. DCT coefficients for every group $u(M \times N \times I)$.

The DC-quantization uses the average value (DCvalue) of the DC coefficients of every group to minimize the DC components as much as possible. We subtract the DCvalue from DC coefficient value for all the bands in the same group. Thus, only the DCvalue for each group is required to be transmitted as side information.

$$
\text { DCvalue }_{u}=\mid \sum_{i=1}^{l} \mathrm{DC} \_ \text {coefficient_band }(i) /\left.l\right|_{\text {Round }}
$$

where $i=1,2, \ldots, /$ is the band index, and $/$ refers to the number of bands per group $u$.

\subsection{Power Allocation and Transmission}

The HyperCast scheme uses an optimal scaling factor $w_{u}$ for the signal $q_{u}$ so that the total distortion on $q_{u}$ is minimized. Scaling the transmitted signal according to [8] provides resilience to channel noise. $w_{u_{i}}$ (the scaling factor for group $u$ at the $i$-th frequency) is given by:

$$
w_{u_{i}}=v_{u_{i}}{ }^{-1 / 4}\left(\sqrt{\frac{P}{\sum_{i=1}^{F} \sqrt{v_{u_{i}}}}}\right)
$$

where $v_{u_{i}}$ is the variance of $q_{u_{i}}$ at the $i$-th frequency, $F$ is the number of frequencies in group $u$, and $P$ is the total transmission power. For each group $q_{u}$, there is only one scaling factor $w_{u}=$ $\operatorname{diag}\left\{w_{u_{1}}, w_{u_{2}}, \ldots, w_{u_{F}}\right\}$ and the signal $q_{u}$ after power allocation is

$$
p_{u}=w_{u} \cdot q_{u} \text {. }
$$

To redistribute energy, the HyperCast scheme protects the weighted signal $p_{u}$ against packet loss through multiplying it by the Hadamard matrix $\mathbf{H}$.

$$
y_{u}=\mathbf{H} \cdot p_{u} .
$$

Traditionally, in the digital communication, FEC and modulation are used. The signal after modulation is then transmitted using traditional OFDM over 802.11 PHY layer. In contrast, the HyperCast scheme transmits signals over OFDM channel without FEC or modulation. For the signal $y_{u}$, every pair of two real samples is combined into one complex symbol as the real and imaginary 
parts of a complex I/Q number as shown in Fig. 5. In addition to the image data, the encoder sends a small amount of metadata containing $m_{u}, D C v a l u e, \mathbf{V}_{u}$ and $\left\{v_{u}\right\}$ to assist the decoder in inverting the received signal. The metadata is transmitted in a traditional way (OFDM in 802.11 PHY with FEC and modulation). Here, binary phase shift keying (BPSK) is used for modulation and a half-rate convolutional code is used for FEC to protect the metadata from channel errors.

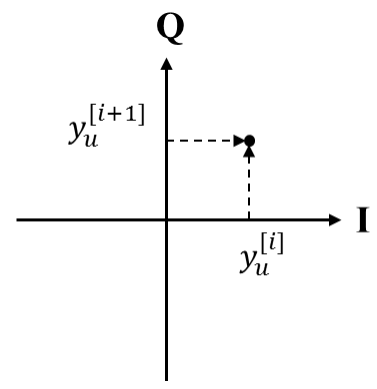

Fig. 5. Mapping data to I/Q components of transmitted OFDM signals.

\subsection{HyperCast Decoder}

\subsubsection{LLSE Decoder}

The signal that arrives at the receiver is $\mathbf{y}^{\prime}=\mathbf{y}+\mathbf{n}$, i.e., we receive a noisy signal

$$
y_{u}{ }^{\prime}=y_{u}+n_{u}
$$

where $n_{u}$ is the random channel noise for group $u$ (additive white Gaussian noise (AWGN)). The LLSE is used to reconstruct the original signal as follows:

$$
q_{u}{ }^{\prime}=\Lambda_{q_{u}} R_{u}{ }^{T}\left(R_{u} \Lambda_{q_{u}} R_{u}^{T}+\Lambda_{n_{u}}\right)^{-1} y_{u}{ }^{\prime}
$$

where $R_{u}=\mathbf{H} \cdot w_{u}$ for group $u, \Lambda_{n_{u}}$ is the covariance matrix of $n_{u}$ and $\Lambda_{q_{u}}$ is a diagonal matrix whose diagonal elements are the variances $\left\{v_{u}\right\}$ of the individual group.

\subsubsection{Inverse Transforms}

After the recovery of signals, a DC-dequantization is performed on each group with the DCvalues. Note that the DCvalues are transmitted as metadata by the encoder. The DCvalue for every group is added to all the DC coefficient of bands in the same group. After that, we reconstruct the group signals by applying the inverse 3D transform. First, we recover the KLT coefficients for group $u$ (i.e., $\left.K_{u}{ }^{\prime}\right)$ by applying the inverse DCT, and then we recover the group signals $\left(g_{u}{ }^{\prime}\right)$ by applying the inverse KLT.

$$
\begin{gathered}
p_{u}^{\prime}[x, y]=\sqrt{\frac{2}{M}} \sqrt{\frac{2}{N}} \sum_{x=0}^{N-1} \sum_{y=0}^{M-1} C_{i} C_{j} d_{u}^{\prime}[i, j] \cos \left[\frac{(2 x+1) i \pi}{2 N}\right] \cos \left[\frac{(2 y+1) j \pi}{2 M}\right], \\
\text { for } 0 \leq x \leq N-1, \text { and } 0 \leq y \leq M-1 . \text { and }
\end{gathered}
$$




$$
C_{f}= \begin{cases}1 / \sqrt{2} & \text { for } f=0 \\ 1 & \text { for } f>0\end{cases}
$$

The result is a set of $t=M \times N$ data values $p^{\prime}[t]$ for each band. All $/$ bands are combined into one group. Once the decoder has obtained the KLT components in $K_{u}{ }^{\prime}$, it can reconstruct the original signal by taking the inverse 1D KLT.

$$
g_{u}{ }^{\prime}=\left(\mathbf{V}_{u}^{\mathbf{T}}\right)^{-1} K_{u}{ }^{\prime}
$$

\subsubsection{Compiling Groups and Band Reordering}

In the final step, we combine the groups $\mathbf{g}^{\prime}=\left\{g_{1}{ }^{\prime}, g_{2}{ }^{\prime}, \ldots, g_{k}{ }^{\prime}\right\}$ into one data set $\mathbf{r}^{\prime}$, and then reorder it and get the final image data. The order indices are transmitted as metadata by the encoder.

$$
\begin{aligned}
& \mathbf{r}^{\prime}=\operatorname{combine}\left(\mathbf{g}^{\prime}\right) \\
& \mathbf{x}^{\prime}=\operatorname{reorder}\left(\mathbf{r}^{\prime}\right)
\end{aligned}
$$

\section{Hyperspectral Band Ordering Optimization}

Every hyperspectral satellite image includes several hundred bands. These bands contain spectral redundancies that needed to be removed. Therefore, optimal band ordering for satellite images has become imperative. The new bands order must minimize the distortion between the neighboring bands to achieve better performance than the original bands order.

In the HyperCast scheme, we propose a band ordering algorithm for the satellite images based on the MSE between image bands and the mean value of each band. The optimal band ordering is the one minimizing the distortion between image bands. In the following subsection, we will briefly review the band ordering algorithms based on the correlation coefficients between image bands. After that, we will introduce the proposed band ordering algorithm.

\subsection{Band Correlation}

The most common approaches to order bands are based on the correlation coefficients between satellite image bands.

In [28], band ordering is applied to satellite images depending on simple correlation between all bands. The band ordering algorithm in [28] is briefly described in three steps.

In the first step, the correlation coefficients between bands are calculated using Eq. (15) (i.e., the correlation coefficient between band $\# 1$ and band $\# 2$ is estimated as $r_{1,2}$, the correlation between band \#1 and band \#3 is estimated as $r_{1,3}$, and so on) to obtain a vector of $\left(\begin{array}{l}\lambda \\ 2\end{array}\right)=\frac{\lambda !}{2 ! \cdot(\lambda-2) !}$ values as $\left[r_{1,2}, r_{1,3}, \ldots, r_{1, \lambda}, r_{2,3}, r_{2,4}, \ldots, r_{2, \lambda}, \ldots, r_{\lambda-1, \lambda}\right]$, where $\lambda$ is the number of bands in the hyperspectral image. 


$$
\operatorname{corr}_{\mathbf{A}, \mathbf{B}}=\frac{\frac{M}{\sum_{j=0 i=0}^{D} \frac{N}{D}}\left(\mathbf{A}_{j . D, i . D}-\overline{\mathbf{A}}\right)\left(\mathbf{B}_{j . D, i . D}-\overline{\mathbf{B}}\right)}{\sqrt{\frac{M}{\sum_{j=0}^{D} \frac{N}{D}}\left(\mathbf{A}_{j=0}-\overline{\mathbf{A}}\right)^{2} \sum_{l=0, i . D}^{\frac{M}{D}} \frac{N}{D}\left(\mathbf{B}_{l . D, k . D}-\overline{\mathbf{B}}\right)^{2}}}
$$

where $M$ is the number of rows and $N$ is the number of columns in the image band, $\overline{\mathbf{A}}$ and $\overline{\mathbf{B}}$ denote the mean values of the bands $\mathbf{A}$ and $\mathbf{B}$ computed for every $D^{\text {th }}$ pixel, respectively. Each $D^{\text {th }}$ pixel in the spatial directions is used for the computation of the correlation coefficients. If $D$ equals 1 , the exact value of the correlation between the two image bands is computed; otherwise Eq. (15) results in a correlation estimate. The larger the value of $D$, the faster the reordering phases of the algorithm; however, at the same time, the estimation accuracy is reduced. In the second step, the absolute values of the correlation vector are sorted in a descending order to determine the bands of the highest correlation. Finally, the bands are interchanged according to the correlation results to achieve the new band ordering for the hyperspectral image.

\subsection{Proposed Band Ordering Algorithm}

A simple and more efficient algorithm for satellite band ordering is proposed. This algorithm is based on the MSE between image bands and the mean value for each band. Different from the correlation coefficient algorithm, the proposed band ordering algorithm uses both the mean value for each band and the difference between bands (i.e., MSE) to find the optimal band ordering. The main idea is to minimize the distortion between bands with low MSE and close mean values. Figure 6 shows an example of all steps of the proposed band ordering algorithm. It can be implemented by Algorithm 1. In the first step, we calculate the mean value for every band in the hyperspectral data $\mathbf{x}$ containing $\lambda$ bands, and then sort the values in matrix $\mathbf{m}$ in a descending order.

$$
m_{i} \leftarrow|\operatorname{mean}(\mathbf{x}(M, N, i))|_{\text {round }}
$$

In the second step, we rearrange the image bands in $\mathbf{x}$ according to the order in $\mathbf{m}$. After that, we divide the mean ordered data into $d$ regions, where $d=\lceil\lambda / s\rceil$ and $s$ is the step size defined by the user as shown in Fig. 6 .

In the third step, we rearrange the image bands in every region according to the minimum mean square error between bands in the same region. We will transform the problem of finding an optimal band ordering for each region into a problem on weighted directed graphs having a known efficient solution. We assume that the nodes of the graph are represented by hyperspectral bands in a certain region, and the weighted values are represented by the mean square errors between bands in the same region as shown in Fig. 6. Finally, we combine the ordered regions into one data matrix $r$ to represent the ordered hyperspectral data. 


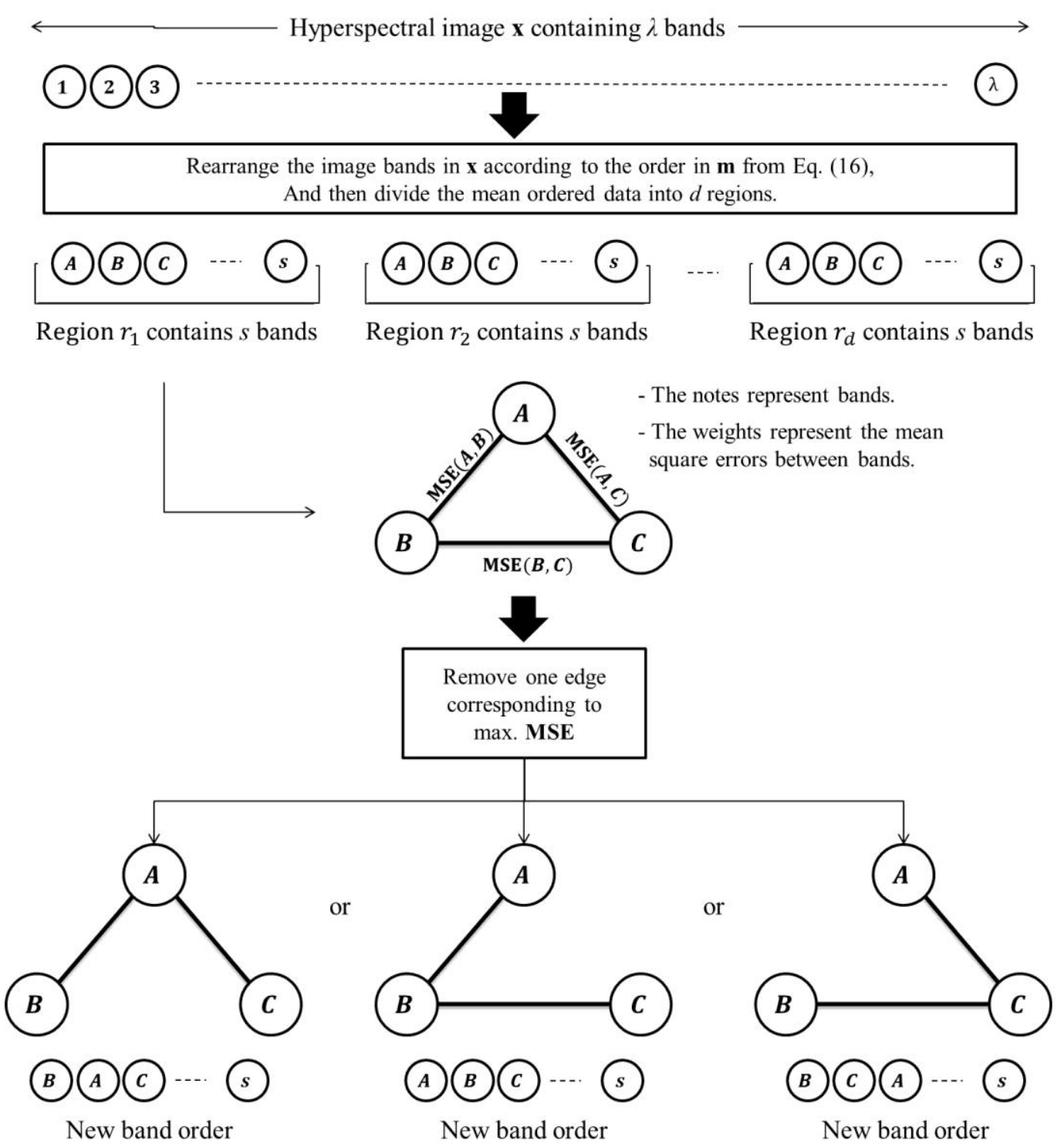

Fig. 6. Example of band ordering for selected three bands $\boldsymbol{A}, \boldsymbol{B}$, and $\boldsymbol{C}$ in the same region.

To optimize the correlation between bands, we divide the image bands into groups using Algorithm 2. Dividing the image bands into groups helps to achieve better compression performance and to reduce the computational complexity of KLT by using a simple clustering approach for each group as shown in Fig. 3 . In the proposed implementation, we divide the image bands into $k$ groups as shown in Fig. 7, where $k=\lceil\lambda / l\rceil, \lambda$ is the number of bands of the hyperspectral image and $l$ is the number of bands of each group (Here, we select $/$ to be four in our implementation, i.e., $I=4$ in Algorithm 2). 
Inputs: Hyperspectral image data $\mathbf{x}$ has $\lambda$ spectral bands, $M \times N$ pixel resolution. Step size $s$.

Output: Ordered hyperspectral image data $\mathbf{r}$.

1: begin

2: $\quad$ for $i=1$ to $\lambda$ do

3: $\quad m_{i} \leftarrow|\operatorname{mean}(\mathbf{x}(M, N, i))|_{\text {round }}$; (Calculate the mean value for every band, and then sort the values in the matrix $\mathbf{m}$ in a descending order).

4: end

5: $\quad \mathbf{y} \leftarrow \operatorname{order}(\mathbf{x}, \mathbf{m})$; (Rearrange the image bands in $\mathbf{x}$ according to the order in $\mathbf{m}$ and write the new ordered image in $\mathbf{y})$.

6: $\quad d=\lceil\lambda / s\rceil ; \quad$ (Divide data $\mathbf{y}$ into $d$ regions as shown in Fig. 6).

7: $\quad$ for $i=1$ to $d$ do

8: $\quad$ for $j=1$ to $s$ do

10: $\quad \forall(j \neq s) e_{i} \leftarrow \operatorname{MSE}(\mathbf{y}\{j\}, \mathbf{y}\{j+1,2, \ldots, s\}) ; \quad$ (Calculate the mean square error for each pair of bands within the same region, and then sort the values in $e$ in a descending order).

11: $\quad r_{i} \leftarrow \operatorname{order}\left(y_{i}, e_{i}\right)$; (Rearrange the image bands in $y_{i}$ (i.e., the region $i$ in the image data $y$ ) according to the order in $e_{i}$ and write the new ordered image in $r_{i}$. Finally, return the ordered hyperspectral image data $r$, where $r$ contains $r_{1}, r_{2}, r_{3}, \ldots, r_{d}$ ).

12: $\quad$ end

13: end

14: end

Algorithm 2: Band Division

Input: Ordered hyperspectral image data r. Number of bands per group I.

Output: Groups of hyperspectral image bands $\mathbf{g}$, where each group contains / bands.

1: begin

2: $\quad k=\lceil\lambda / l\rceil ; \quad$ (Calculate the number of groups per image in $k$ ). 


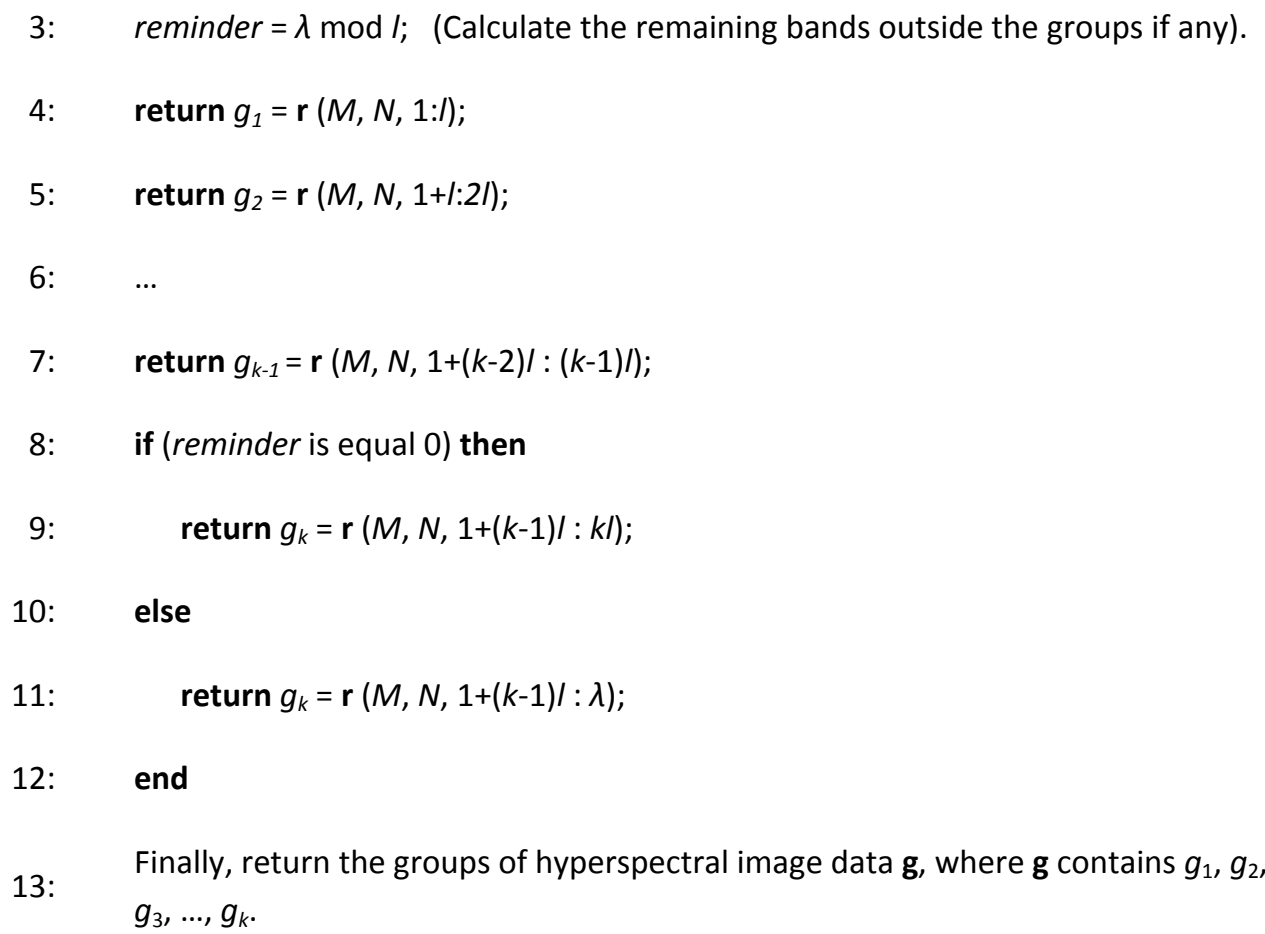

14: end

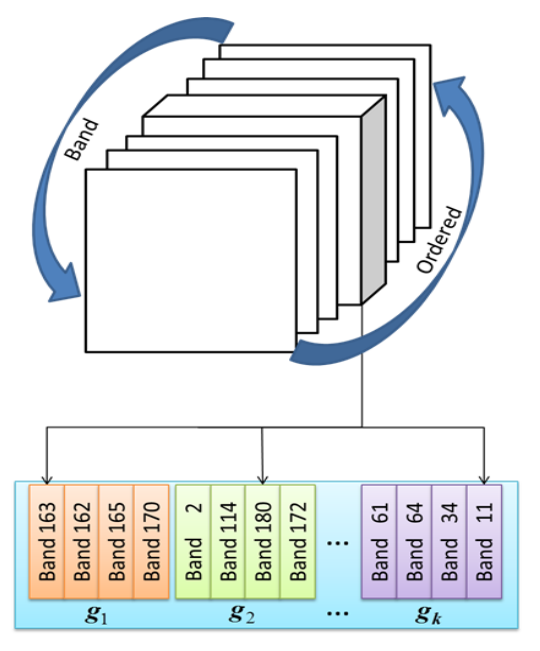

Fig. 7. Hyperspectral band ordering and grouping.

\section{Evaluation and Results}

\subsection{Dataset}

The proposed HyperCast scheme has been tested for several hyperspectral images of the Airborne Visible/Infrared Imaging Spectrometer (AVIRIS) sensor, which was developed by the NASA Jet Propulsion Laboratory in 1987. This sensor provides spectral images with 224 contiguous bands covering the spectral range from $0.41 \mu \mathrm{m}$ to $2.45 \mu \mathrm{m}$. The dataset provided by CCSDS 
(http://www.ccsds.org) includes seven uncalibrated radiance hyperspectral images, acquired over Yellowstone, WY in 2006, namely, YellowstoneUn_Sc0, YellowstoneUn_Sc03, YellowstoneUn_Sc10, YellowstoneUn_Sc11, YellowstoneUn_Sc18, Maine_Sc10, and Hawaii_Sc01. Each image has 224 spectral bands, $680 \times 512$ pixel resolution, and each pixel is represented by 16 bits per pixel per band (bpppb) except for Maine and Hawaii that have $12 \mathrm{bpppb}$. Hawaii has $614 \times 512$ pixel resolution. All images are publicly available for download (http://aviris.jpl.nasa.gov/). False-color images of the selected dataset are shown in Fig. 8. A false-color image (implemented in ENVI4.5 (ITT VIS 2008) http://www.ittvis.com/envi/) depends on radiance or reflectance from three channels; red, green and blue with wavelengths of about $650 \mathrm{~nm}, 510 \mathrm{~nm}$ and $475 \mathrm{~nm}$, respectively. In hyperspectral images, bands 125,70 , and 30 represent red, green, and blue wavelengths, respectively.

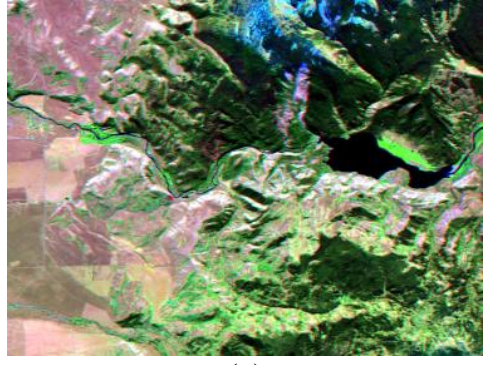

(a)

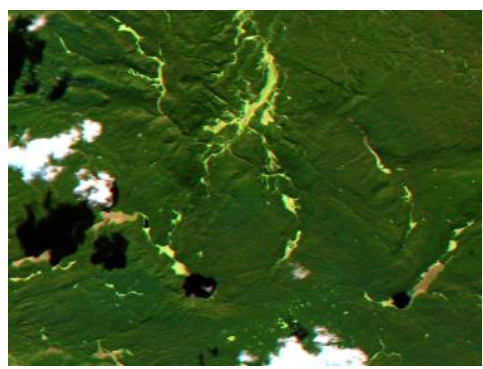

(d)

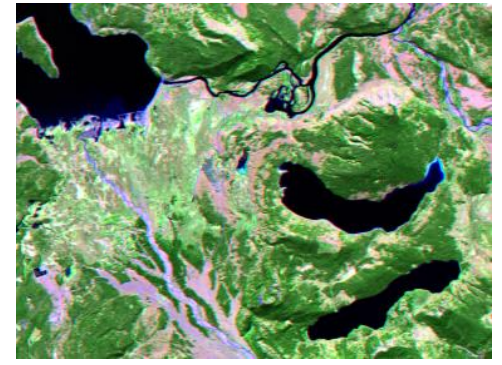

(b)

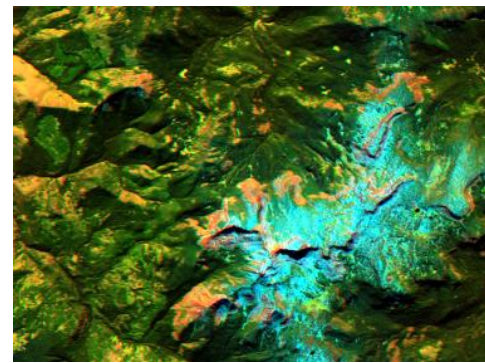

(e)

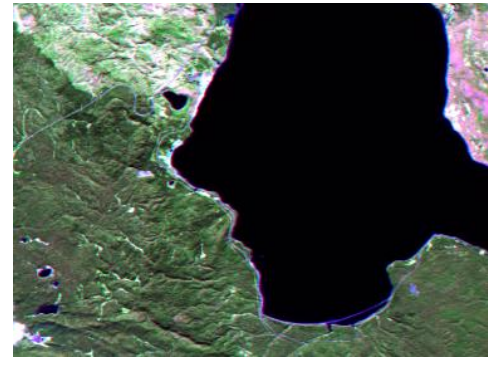

(c)

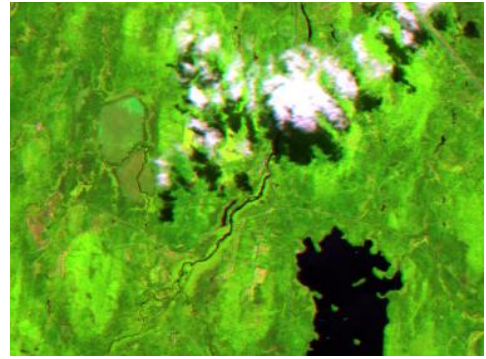

(f)

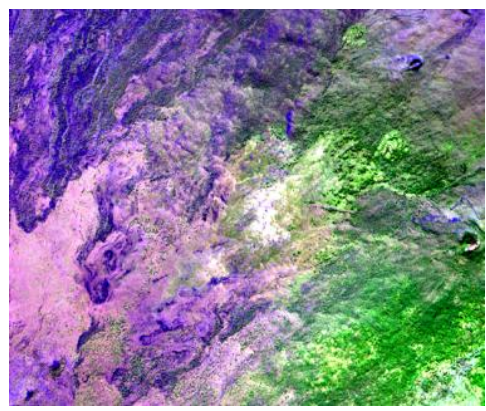

(g)

\subsection{Evaluation of Band Ordering}

We evaluate the performance of the proposed band ordering algorithm and show that it improves the image quality compared with the correlation coefficients algorithm and without band ordering under broadcasting channels. In Fig. 9, two other schemes are compared here, HyperCast that implementing correlation band ordering and HyperCast with that does not implement band ordering. 
Quality is computed comparing the original image $\mathbf{x}(M, N, \lambda)$ with the recovered image $\mathbf{x}^{\prime}(M$, $N, \lambda)$. We evaluate all methods using the peak signal-to-noise ratio (PSNR),

$$
\begin{gathered}
\operatorname{PSNR}_{(\mathrm{dB})}=10 \cdot \log _{10}\left(\frac{L^{2}}{\mathrm{MSE}}\right)(\mathrm{dB}) \\
\mathrm{MSE}=\frac{1}{M \cdot N \cdot \lambda} \sum_{i=1}^{M} \sum_{j=1}^{N} \sum_{k=1}^{\lambda}\left[\mathbf{x}(i, j, k)-\mathbf{x}^{\prime}(i, j, k)\right]^{2}
\end{gathered}
$$

where $\lambda$ is the number of hyperspectral image bands, and $L$ is the maximum possible pixel value of the hyperspectral image (i.e., $L=2^{B}-1$, where $B$ is the bit depth). Typical values of the PSNR in lossy hyperspectral image compression are between 50 and $80 \mathrm{~dB}$, provided the bit depth is 16 bits, where higher is better.

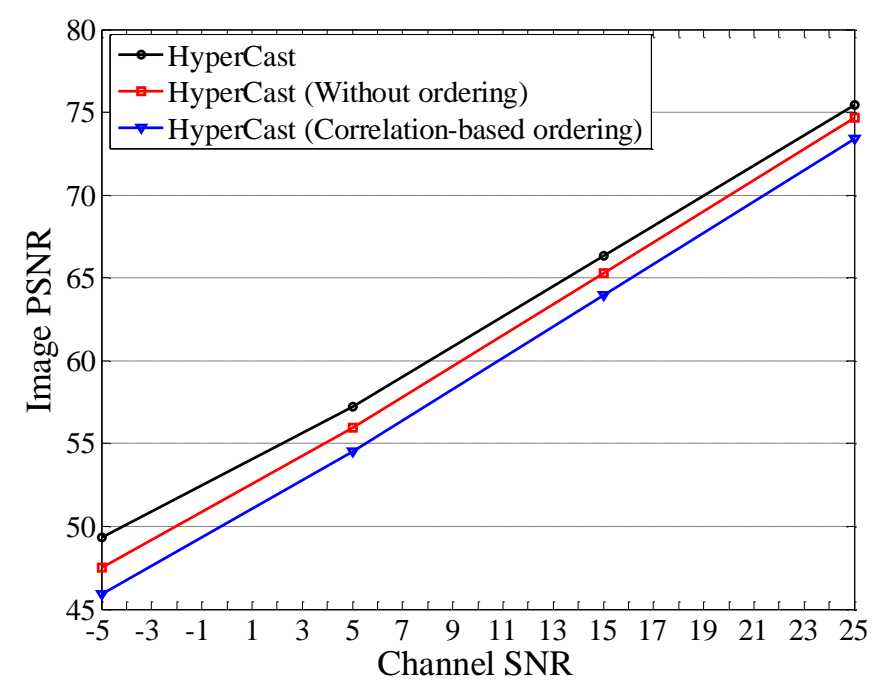

Fig. 9. HyperCast band ordering performance.

It is shown in Fig. 9 that the proposed band ordering algorithm achieves a better performance as compared with the cases of correlation-based band ordering and no band ordering. By removing the band ordering process, there is $1.30 \mathrm{~dB}$ loss on average PSNR. The consumed time of the proposed band ordering algorithm is given in Table 1. The test machine has an Intel(R) Xeon $(R)$ CPU E3-1230 V2 @ 3.30GHz 3.70GHz, 32GB internal memory and Microsoft Windows 8.1 Enterprise 64-bit. All results shown in Table 1 are measured in seconds.

Table 1: Consumed time in second for the proposed band ordering algorithm.

Hyperspectral image containing 224 bands. 


\begin{tabular}{|c|c|c|c|c|c|c|c|}
\hline Image & Image & Image & Image & Image & Image & Image & Average \\
1 & 2 & 3 & 4 & 5 & 6 & 7 & \\
\hline $95.09 \mathrm{~s}$ & $95.52 \mathrm{~s}$ & $97.64 \mathrm{~s}$ & $96.57 \mathrm{~s}$ & $95.52 \mathrm{~s}$ & $95.09 \mathrm{~s}$ & $88.64 \mathrm{~s}$ & $\mathbf{9 4 . 8 6 s}$ \\
\hline
\end{tabular}

\subsection{Evaluation of HyperCast}

As presented before in Section 2, LineCast is the up-to-date satellite image delivery scheme. However, the LineCast scheme does not work well with satellite images containing more than one band, because there is no spectral correlation between bands. In the LineCast scheme, every scanned line of an image is compressed by the transform-domain scalar modulo quantization without prediction. We have implemented the LineCast scheme [9] for every band, separately, in the selected hyperspectral images.

The SoftCast differs from the LineCast because of using a 3D transformation to remove both spectral and spatial redundancies form the satellite image data. The full band is transformed in the proposed implementation. We compare HyperCast with the 3D version of SoftCast based on 3D DCT in [8].

The JPEG2000 with PCA in the spectral decorrelator is used in digital source coding. The 3D hyperspectral image data is first decorrelated with PCA, and then a 2D DWT with 5 level decomposition is used in spatial decorrelation. In the DWT, the $9 / 7$ filter has been used and the reference software BOI [29] has been used for implementation. The experimental results for digital separable source-channel coding (i.e., JPEG2000 with PCA for source coding and different combinations of FEC rates and modulation methods for channel coding) are listed in Table 2. For HyperCast, SoftCast and LineCast, there is no bit rate but only channel symbol rate. We assume that the channel noise is AWGN and the channel bandwidth is equal to the source bandwidth. All frameworks consume the same bandwidth and transmission power. We have implemented all frameworks using MATLAB R2014a.

Table 2: Combinations of source rates, FEC rates and modulation methods in JPEG2000.

\begin{tabular}{|c|c|c|c|c|c|c|c|c|}
\hline & \multirow{2}{*}{$\begin{array}{c}\text { Source } \\
\text { REC + Modulation }\end{array}$} & \multicolumn{6}{|c|}{ Reconstruction PSNR without channel noise (dB) } \\
\cline { 5 - 9 } & \begin{tabular}{c} 
(bpppb) \\
\cline { 5 - 9 }
\end{tabular} & $\begin{array}{c}\text { Image } \\
1\end{array}$ & $\begin{array}{c}\text { Image } \\
2\end{array}$ & $\begin{array}{c}\text { Image } \\
3\end{array}$ & $\begin{array}{c}\text { Image } \\
4\end{array}$ & $\begin{array}{c}\text { Image } \\
5\end{array}$ & $\begin{array}{c}\text { Image } \\
6\end{array}$ & $\begin{array}{c}\text { Image } \\
7\end{array}$ \\
\hline $1 / 2$ FEC + BPSK & 0.25 & 49.488 & 50.216 & 56.077 & 53.751 & 48.101 & 53.759 & 56.298 \\
\hline $1 / 2$ FEC + QPSK & 0.5 & 52.060 & 52.544 & 59.250 & 56.292 & 50.886 & 56.811 & 58.309 \\
\hline $3 / 4$ FEC + QPSK & 0.75 & 54.338 & 54.775 & 62.138 & 58.341 & 52.906 & 59.177 & 60.165 \\
\hline $1 / 2$ FEC + 16QAM & 1.0 & 55.989 & 56.450 & 64.930 & 60.281 & 54.908 & 61.275 & 61.669 \\
\hline
\end{tabular}




\begin{tabular}{|c|c|c|c|c|c|c|c|c|}
\hline 3/4 FEC + 16QAM & 1.5 & 59.585 & 60.197 & 69.943 & 63.963 & 58.533 & 65.188 & 64.775 \\
\hline 2/3 FEC + 64QAM & 2.0 & 62.943 & 63.730 & 74.615 & 67.438 & 62.047 & 68.499 & 67.847 \\
\hline 3/4 FEC + 64QAM & 2.25 & 64.495 & 65.406 & 76.806 & 68.915 & 63.557 & 70.032 & 69.167 \\
\hline
\end{tabular}

First, we evaluate the performance of the HyperCast scheme over unicast test among LineCast, SoftCast, and the conventional framework (i.e., JPEG2000 with different combinations of FEC rates and modulation methods). The experimental result is given in Fig. 10. In this test the channel SNR is between $5 \mathrm{~dB}$ and $25 \mathrm{~dB}$. The conventional framework is assumed to be able to choose the best combinations of FEC and the modulation methods recommended by 802.11 according to the channel SNR, to get maximal bitrate for JPEG2000 codec. Both the encoder the decoder is assumed to know the channel SNR. The proposed HyperCast delivers a PSNR gain up to $6.71 \mathrm{~dB}$, $3.23 \mathrm{~dB}$, and 4.99dB over LineCast, SoftCast-3D, and JPEG2000 based framework, respectively.

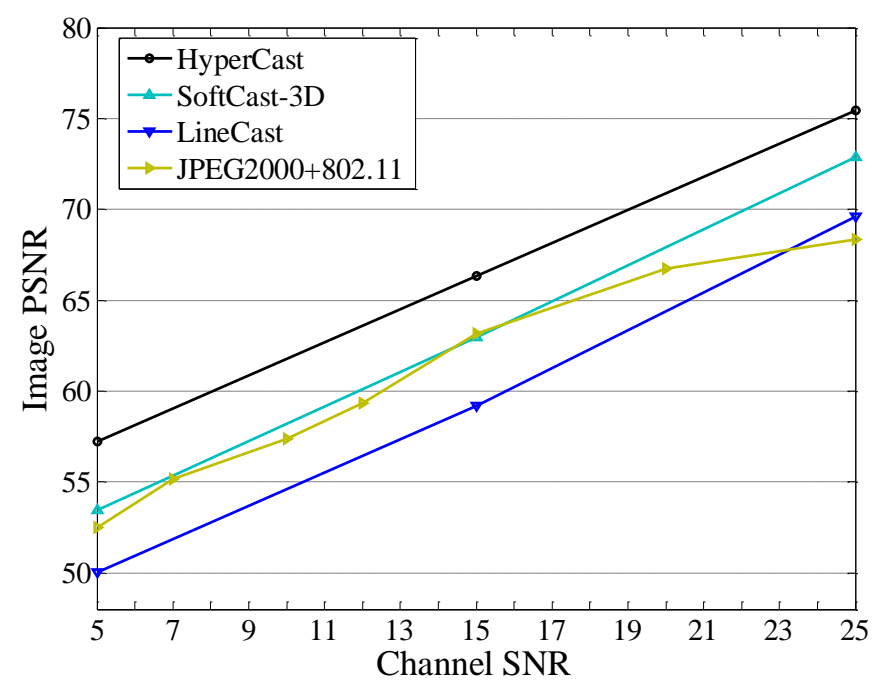

Fig. 10. Unicast performance comparison.

Second, we evaluate the performance of the HyperCast scheme over broadcasting channels and compare it to all the above frameworks. The PSNR of the reconstructed images of each scheme for different channel SNR values between $O d B$ and $25 \mathrm{~dB}$ are given in Fig. 11 . We can see that the proposed HyperCast scheme performs better than the other schemes for broadcasting of hyperspectral satellite images. HyperCast delivers in broadcasting a PSNR gain up to $1.30 \mathrm{~dB}, 6.98 \mathrm{~dB}$, $3.48 \mathrm{~dB}$ and $6.14 \mathrm{~dB}$ over HyperCast that does not implement band ordering, LineCast, SoftCast-3D, and conventional framework, respectively. Note that the result in Fig. 11 does not mean HyperCast scheme can outperform JPEG2000 in compression efficiency. This method is satellite image coding standards, while the proposed is a wireless satellite image transmission framework. The compression methods have very high compression efficiency but the bitstream is not very robust to 
error. This why the bitstreams need additional FEC bits for protection. However, the proposed scheme is robust to channel noise. Thus, it can skip FEC, and is able to achieve high transmission efficiency.

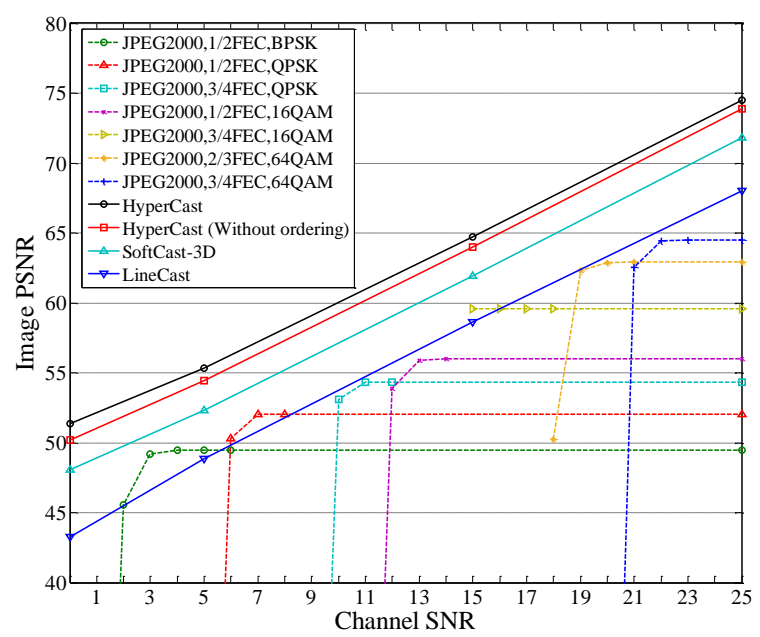

(a)

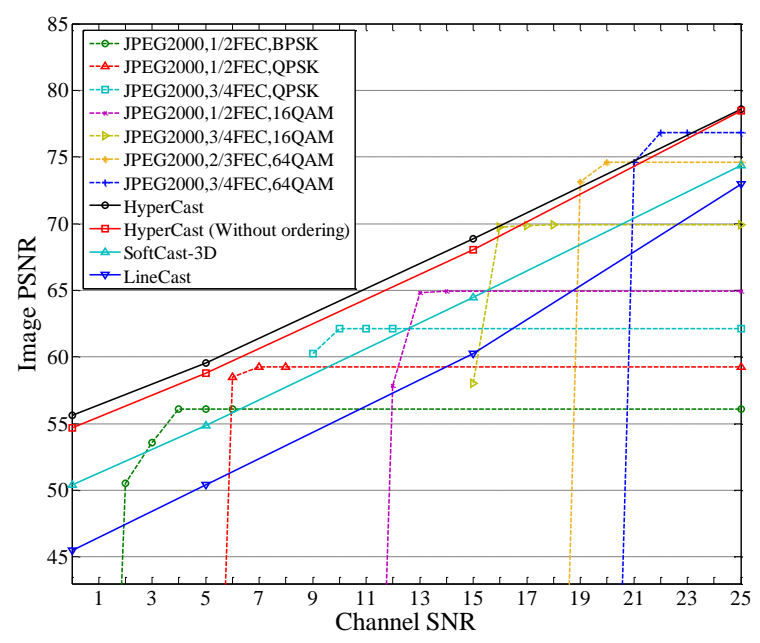

(c)

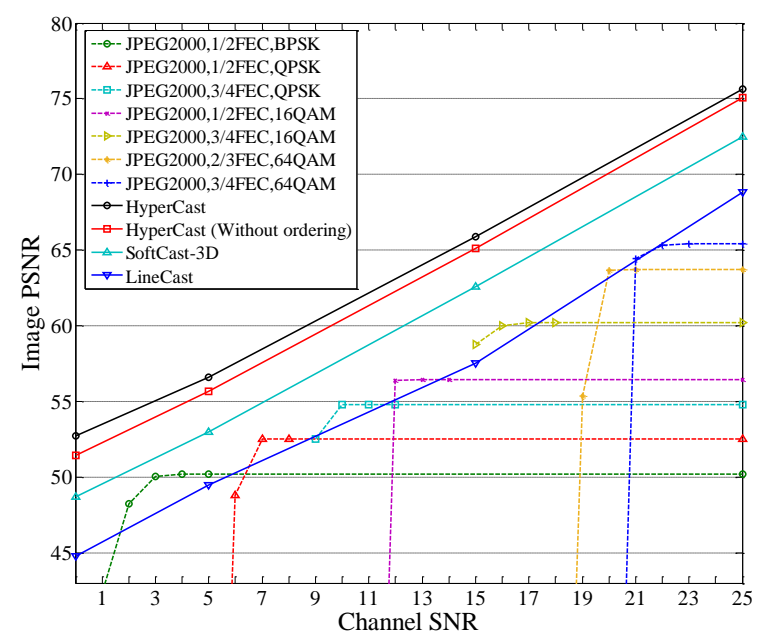

(b)

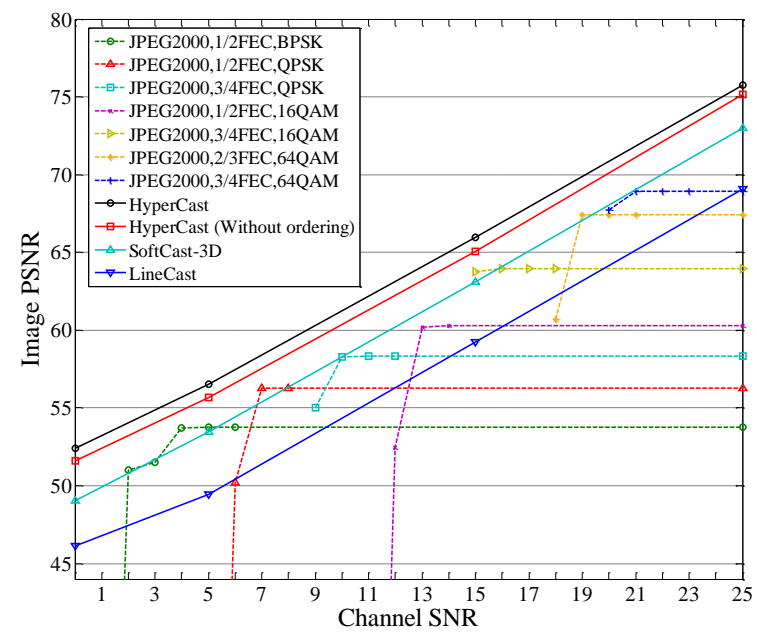

(d) 


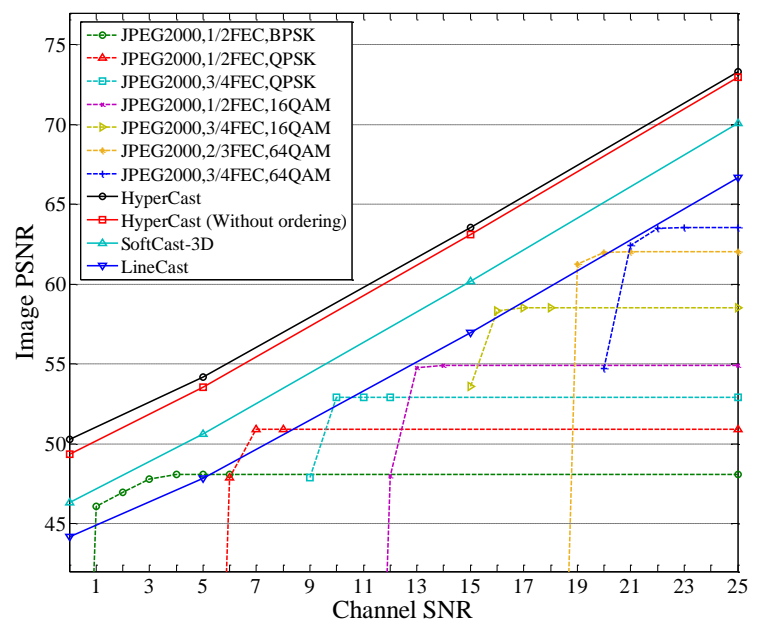

(e)

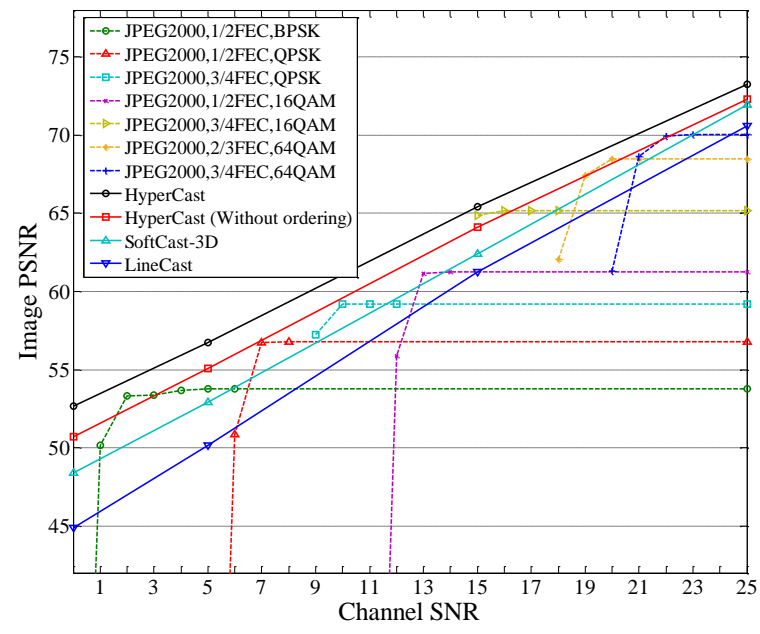

(f)

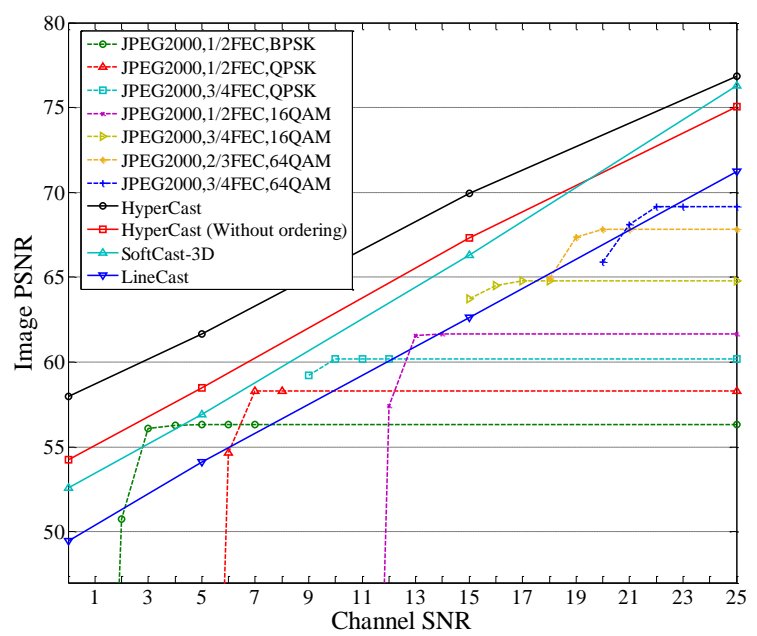

(g)

Fig. 11. Broadcasting performance comparison on different AVIRIS images. (a) Image 1, (b) Image 2, (c) Image 3, (d) Image 4, (e) Image 5, (f) Image 6, and (g) Image 7.

In Fig. 11, we notice also that the separable source-channel coding (i.e., JPEG2000 with FEC and modulation) shows cliff effect for all seven conventional transmission approaches. For example, the approach 'JPEG2000, 3/4FEC, QPSK' performs well, when the channel SNR is between $10 \mathrm{~dB}$ and $12 \mathrm{~dB}$, but it is not good when the channel SNR is out of this range. There is no improvement in the image quality in terms of SNR by more than $12 \mathrm{~dB}$. In contrast, the HyperCast, SoftCast, and LineCast schemes avoid the annoying cliff effect. The reconstruction PSNR increases linearly with the increasing of the SNR, and vice versa.

\subsection{Visual Quality}

The visual quality comparison among HyperCast, LineCast, SoftCast-3D, and JPEG2000 at 0.25bpppb with $1 / 2$ FEC and BPSK is shown in Fig. 12. The channel SNR is set to $5 \mathrm{~dB}$. Image 1 : YellowstoneUn_Sc0, band 77 is selected. HyperCast shows better visual quality than the others. 
These results ensure the effectiveness of the proposed HyperCast scheme, and it can be further improved in the future.

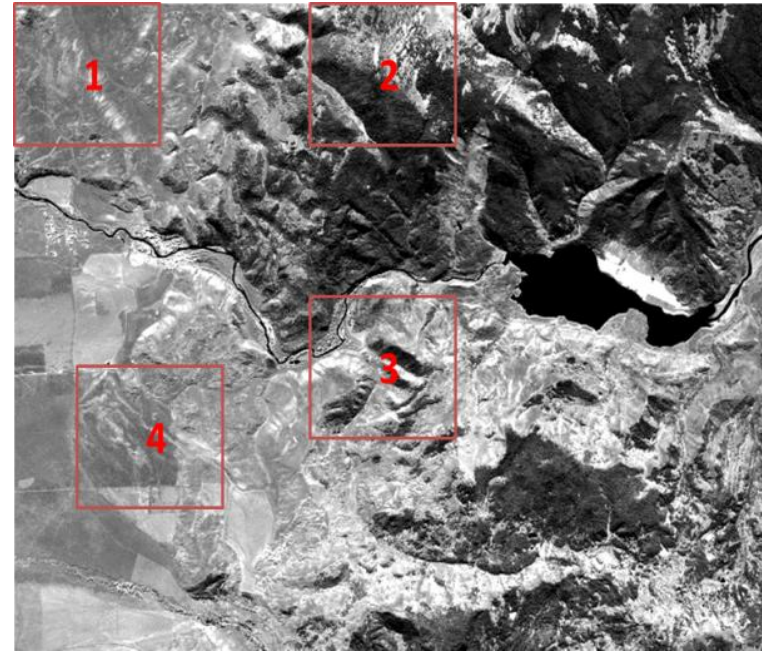

(a)

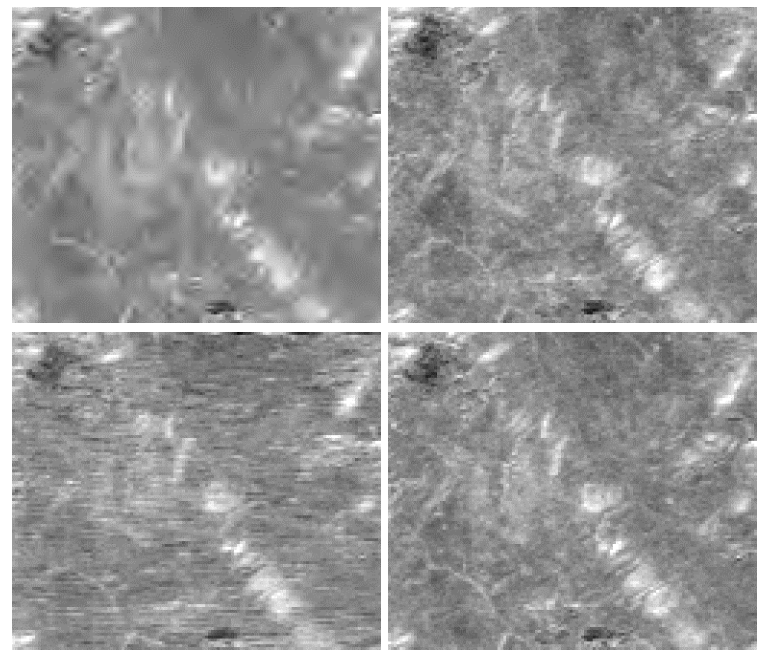

(c)

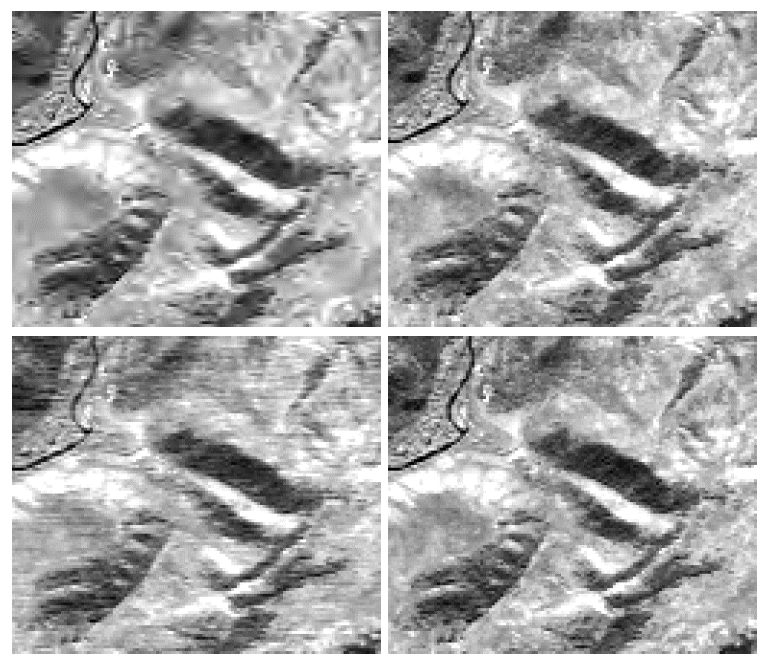

(e)

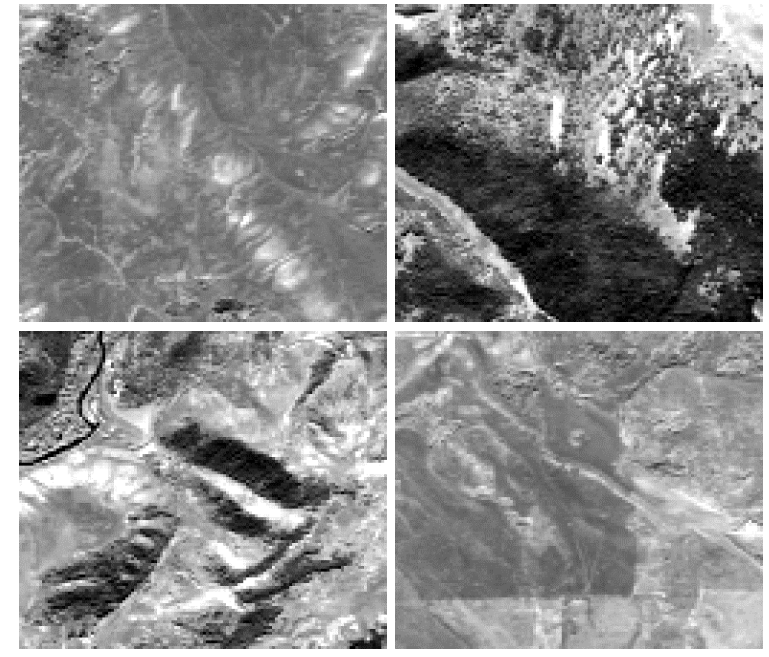

(b)

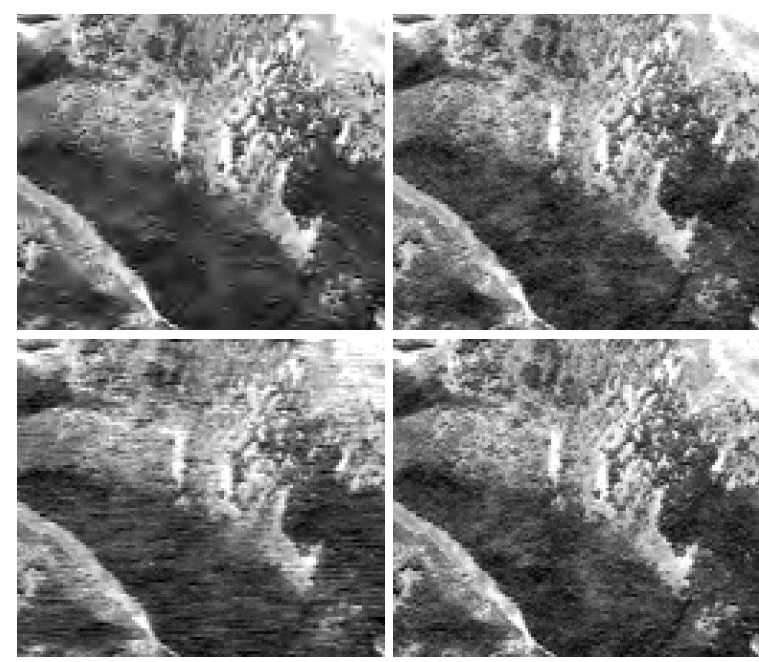

(d)

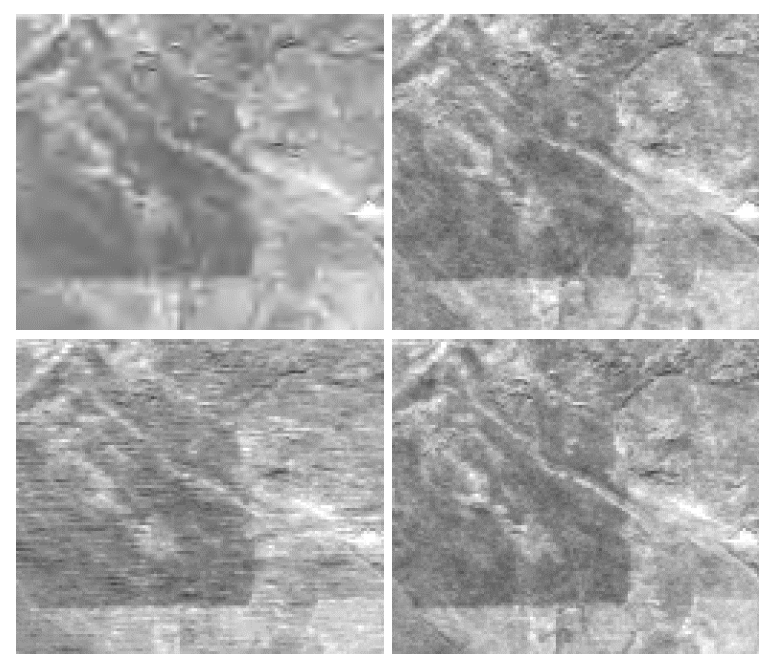

(f) 
Fig. 12. Visual quality comparison at $\mathrm{SNR}=5 \mathrm{~dB}$. (a) Image 1: YellowstoneUn_Sc0 band 77, Full size. (b) Original four rectangular regions. (c)-(f) Reconstructed four rectangular regions, where the four results from left to right, top to bottom are: JPEG2000 at $0.25 \mathrm{bpppb}$ with 1/2 FEC and BPSK, SoftCast-3D, LineCast and HyperCast with PSNRs $43.20725 \mathrm{~dB}, 44.36997 \mathrm{~dB}, 40.68327 \mathrm{~dB}$, and $46.21833 \mathrm{~dB}$, respectively.

\subsection{Encoding Complexity}

At the encoder of the proposed scheme, we divided the hyperspectral band into groups, and after that, a low-complexity KLT is used for spectral decorrelation. Therefore, the low complexity has been achieved during the encoding process. Figure 13 shows the average encoding time per hyperspectral image using different schemes (JPEG2000 with FEC and modulation, SoftCast-3D, LineCast and HyperCast). The test machine has an Intel(R) Xeon(R) CPU E3-1230 V2 @ 3.30GHz 3.70GHz, 32GB internal memory and Microsoft Windows 8.1 Enterprise 64-bit. All the encoding time results shown in Fig. 13 are measured in seconds.

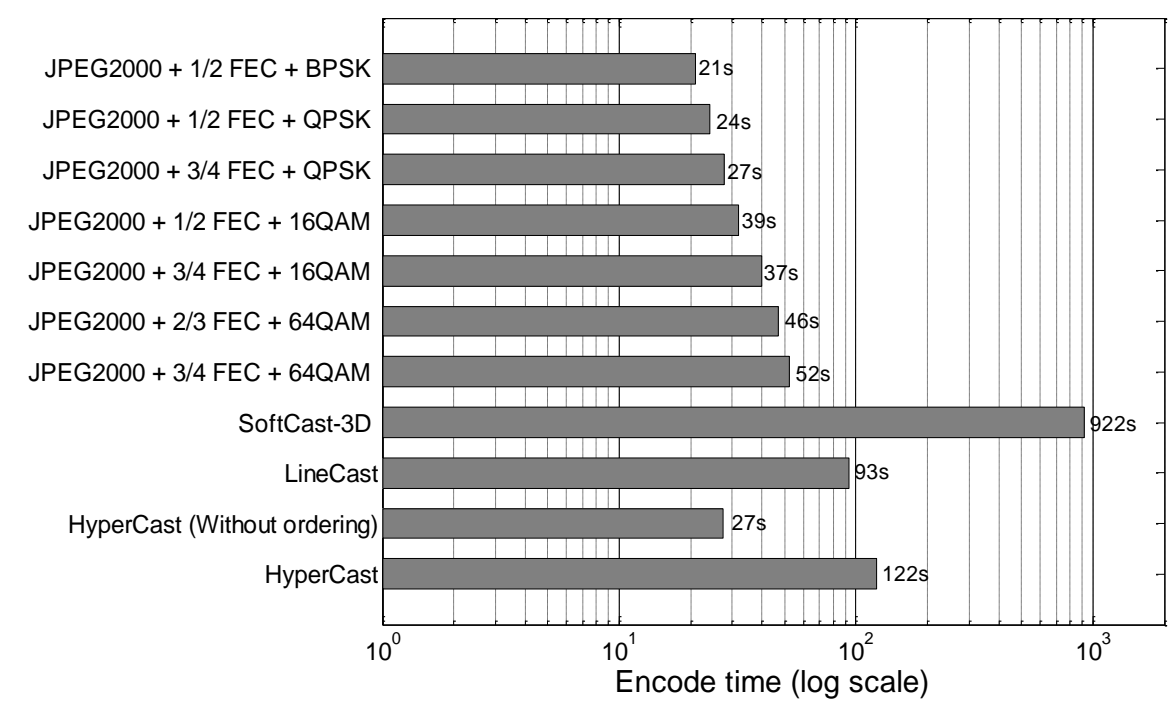

Fig. 13. Encoding complexity of the selected schemes.

As shown in Fig. 13, in the case of JPEG2000 with FEC and modulation, the encoding time becomes longer, when the bitrate increases. The HyperCast scheme without band ordering has a less encoding time than JPEG2000, when the FEC is $1 / 2$ and the modulation is 16 QAM, whereas it is approximately equal to JPEG2000, when the FEC is $3 / 4$ and the modulation is QPSK. The proposed scheme has neither FEC nor modulation at all. In the HyperCast scheme with a band ordering step, we add the band ordering time to the encoding time. It is still less than the time of the state-of-theart 3D scheme (i.e., SoftCast-3D). The SoftCast-3D is the longest encoding time due to the full band size of the 3D DCT used in the encoder process.

\section{Conclusions}

This paper presents a HyperCast scheme, which is a novel scheme for hyperspectral satellite image broadcasting. The HyperCast first presents a new band ordering algorithm for satellite images. 
Furthermore, we also divide image bands into groups to optimize the correlation estimation between bands. The HyperCast scheme takes advantage of the spectral redundancies in satellite images by the use of a simple and low-complexity Karhunen-Loève transform (KLT) as a spectral decorrelation transform, followed by a 2D discrete cosine transform (DCT) for spatial decorrelation. The HyperCast scheme avoids the annoying cliff effect found in digital broadcasting schemes by using a linear transform between the transmitted image and the original pixels' luminance. Experimental results from the selected hyperspectral datasets demonstrate that the proposed HyperCast scheme is more effective than the other schemes.

\section{Acknowledgement}

This work was supported in part by the National Science Foundation of China (NSFC) under grants 61472101, 61631017 and 61390513, the Major State Basic Research Development Program of China (973 Program 2015CB351804), and the National High Technology Research and Development Program of China (863 Program 2015AA015903). The authors would like to thank Dr. Ibrahim Omara for his support in this work and also the anonymous reviewers for their valuable comments that greatly improved this paper.

\section{References}

[1] I. Blanes, E. Magli, and J. Serra-Sagrista, "A tutorial on image compression for optical space imaging systems," Geoscience and Remote Sensing Magazine, IEEE, vol. 2, no. 3, pp. 8-26, 2014.

[2] M. Johnson, K. Freeman, R. Gilstrap, and R. Beck, "Networking technologies enable advances in earth science," Computer Networks, vol. 46, no. 3, pp. 423-435, 2004.

[3] M. D. Crowley, W. Chen, E. J. Sukalac, X. Sun, P. L. Coronado, and G.-Q. Zhang, "Visualization of remote hyperspectral image data using google earth," in Geoscience and Remote Sensing Symposium, 2006. IGARSS 2006. IEEE International Conference on. IEEE, 2006, pp. 907-910.

[4] C. E. Shannon, "A mathematical theory of communication," ACM SIGMOBILE Mobile Computing and Communications Review, vol. 5, no. 1, pp. 3-55, 2001.

[5] C. E. Shannon et al., "Two-way communication channels," in Proc. 4th Berkeley Symp. Math. Stat. Prob, vol. 1. Citeseer, 1961, pp. 611-644.

[6] Q. Xu, V. Stankovic, and Z. Xiong, "Distributed joint source-channel coding of video using raptor codes," Selected areas in communications, IEEE Journal on, vol. 25, no. 4, pp. 851-861, 2007.

[7] A. D. Liveris, Z. Xiong, and C. N. Georghiades, "Joint source-channel coding of binary sources with side information at the decoder using ira codes," in Multimedia Signal Processing, 2002 IEEE Workshop on. IEEE, 2002, pp. 53-56.

[8] S. Jakubczak and D. Katabi, "A cross-layer design for scalable mobile video," in Proceedings of the 17th annual international conference on Mobile computing and networking. ACM, 2011, pp. 289-300.

[9] F. Wu, X. Peng, and J. Xu, "Linecast: Line-based distributed coding and transmission for broadcasting satellite images," Image Processing, IEEE Transactions on, vol. 23, no. 3, pp. 1015-1027, 2014.

[10] I. Blanes and J. Serra-Sagristà, "Clustered reversible-klt for progressive lossy-to-lossless 3d image coding," in Data Compression Conference, 2009. DCC'09. IEEE, 2009, pp. 233-242. 
[11] D. Taubman and M. Marcellin, JPEG2000 Image Compression Fundamentals, Standards and Practice: Image Compression Fundamentals, Standards and Practice. Springer Science \& Business Media, 2012, vol. 642.

[12] Q. Du and J. E. Fowler, "Hyperspectral image compression using jpeg2000 and principal component analysis," Geoscience and Remote Sensing Letters, IEEE, vol. 4, no. 2, pp. 201-205, 2007.

[13] A. Hagag, M. Amin, and F. E. A. El-Samie, "Simultaneous denoising and compression of multispectral images," Journal of Applied Remote Sensing, vol. 7, no. 1, pp. 073511-073511, 2013.

[14] G. Carvajal, B. Penna, E. Magli et al., "Unified lossy and near-lossless hyperspectral image compression based on jpeg 2000," IEEE Geosci. Remote Sens. Lett, vol. 5, no. 4, pp. 593-597, 2008.

[15] M. M. Ghandi and M. Ghanbari, "Layered h. 264 video transmission with hierarchical qam," Journal of Visual Communication and Image Representation, vol. 17, no. 2, pp. 451-466, 2006.

[16] Z. Reznic, M. Feder, and S. Freundlich, "Apparatus and method for applying unequal error protection during wireless video transmission," Aug. 23 2011, uS Patent 8,006,168.

[17] K. Ramchandran, A. Ortega, K. M. Uz, and M. Vetterli, "Multiresolution broadcast for digital hdtv using joint source/channel coding," Selected Areas in Communications, IEEE Journal on, vol. 11, no. 1, pp. 6-23, 1993.

[18] H. Schwarz, D. Marpe, and T. Wiegand, "Overview of the scalable video coding extension of the h. 264/avc standard," Circuits and Systems for Video Technology, IEEE Transactions on, vol. 17, no. 9, pp. 1103-1120, 2007.

[19] T. Kratochvl, "Hierarchical modulation in dvb-t/h mobile tv transmission," in Multi-Carrier Systems \& Solutions 2009. Springer, 2009, pp. 333-341.

[20] Consultative Committee for Space Data Systems (CCSDS), Image Data Compres-sion CCSDS 122.0-B-1, Nov. 2005. [Online]. Available: http://public.ccsds.org/publications/archive/122x0b1c3.pdf

[21] X. Fan, F. Wu, D. Zhao, and O. C. Au, "Distributed wireless visual communication with power distortion optimization," Circuits and Systems for Video Technology, IEEE Transactions on, vol. 23, no. 6, pp. 10401053, 2013.

[22] B. Penna, T. Tillo, E. Magli, and G. Olmo, "Transform coding techniques for lossy hyperspectral data compression," Geoscience and Remote Sensing, IEEE Transactions on, vol. 45, no. 5, pp. 1408-1421, 2007.

[23] I. Blanes, J. Serra-Sagrista, M. W. Marcellin, and J. Bartrina-Rapesta, "Divide-and-conquer strategies for hyperspectral image processing: a review of their benefits and advantages," Signal Processing Magazine, IEEE, vol. 29, no. 3, pp. 71-81, 2012.

[24] B. Penna, T. Tillo, E. Magli, and G. Olmo, "A new low complexity klt for lossy hyperspectral data compression," in Proceedings of the International Geoscience and Remote Sensing Symposium, vol. 7, no. 06, 2006, pp. 3508-3511.

[25] J. A. Saghri, S. Schroeder, and A. G. Tescher, "Adaptive two-stage karhunen-loeve-transform scheme for spectral decorrelation in hyperspectral bandwidth compression," Optical Engineering, vol. 49, no. 5, pp. 057001-057001, 2010. 
[26] I. Blanes and J. Serra-Sagristà, "Pairwise orthogonal transform for spectral image coding," Geoscience and Remote Sensing, IEEE Transactions on, vol. 49, no. 3, pp. 961-972, 2011.

[27] Q. Du, W. Zhu, H. Yang, and J.E. Fowler, "Segmented principal component analysis for parallel compression of hyperspectral imagery," Geoscience and Remote Sensing Letters, IEEE, vol. 6, no. 4, pp. 713-717, 2009.

[28] A. Hagag, M. Amin, and F. E. A. El-Samie, "Multispectral image compression with band ordering and wavelet transforms," Signal, Image and Video Processing, vol. 9, no. 4, pp. 769-778, 2015.

[29] F. Aulí-Llinàs, “Boi codec," 2014. [Online]. Available: http://www.deic.uab.cat/ francesc/software/boi/ 


\section{Author Biographies}

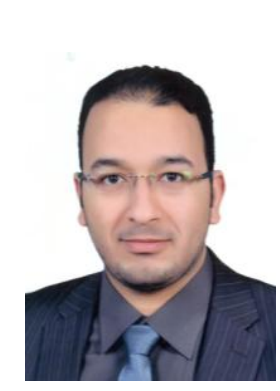

Ahmed Hagag received the B.Sc. (Honors) in pure mathematics and computer science from the Faculty of Science, Menoufia University, Egypt, in 2008, and he received his M.Sc. in computer science form the same university in 2013. He joined the teaching staff of the Faculty of Computer and Information Technology, Egyptian E-Learning University, Cairo, Egypt, in 2009. Currently, he is pursuing the Ph.D. degree with the Department of Computer Science at the School of Computer Science and Technology, Harbin Institute of Technology (HIT). His research interests are compression, classification, de-nosing, and wireless communication for satellite multispectral and hyperspectral images.

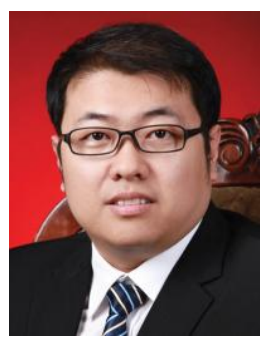

Xiaopeng Fan received the B.S. and M.S. degrees from the Harbin Institute of Technology (HIT), China, in 2001 and 2003 respectively, and the Ph.D. degree from Hong Kong University of Science and Technology (HKUST) in 2009. In 2009, he joined the Department of Computer Science, Harbin Institute of Technology (HIT), where he is currently a Professor. From 2003 to 2005, he worked with Intel China Software Laboratory (ICSL) as Software Engineer. His current research interests are in image/video processing and wireless communication. He has authored or co-authored over 80 technical journal and conference papers. His transform and error resilience algorithms are accepted into the China AVS standard. He served as associate editor of IEEE AVS standard from 2012. He is a recipient of the New Century Excellent Talents in University

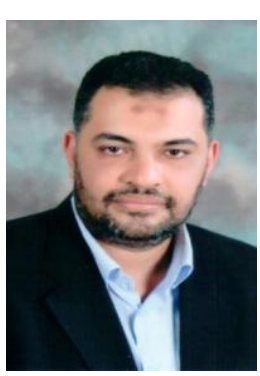

(NCET) Program of China and the MSRA 'StarTrack' Visiting Young Faculty Program in 2011.

Fathi E. Abd El-Samie received the B.Sc. (Honors), M.Sc., and Ph.D. degrees from Menoufia University, Menouf, Egypt, in 1998, 2001, and 2005, respectively. He is a Professor at the Department of Electronics and Electrical Communications, Faculty of Electronic Engineering, Menoufia University, Egypt. He is a coauthor of several papers and textbooks. His current research interests include image enhancement, image restoration, image interpolation, super-resolution reconstruction of images, data hiding, multimedia communications, medical image processing, optical signal processing, and digital communications. Dr. Abd El-Samie was a recipient of the Most Cited Paper Award from the Digital Signal Processing journal in 2008. 